Meta

Journal des traducteurs

Translators' Journal

\title{
La traduction des éponymes médicaux banalisés de langue anglaise
}

\section{Henri Van Hoof}

Volume 46, numéro 1, mars 2001

Traduction médicale et documentation / Medical translation and documentation

URI : https://id.erudit.org/iderudit/004549ar

DOI : https://doi.org/10.7202/004549ar

Aller au sommaire du numéro

Éditeur(s)

Les Presses de l'Université de Montréal

ISSN

0026-0452 (imprimé)

1492-1421 (numérique)

Découvrir la revue

Citer cet article

Van Hoof, H. (2001). La traduction des éponymes médicaux banalisés de langue anglaise. Meta, 46(1), 82-91. https://doi.org/10.7202/004549ar d'utilisation que vous pouvez consulter en ligne. 


\title{
La traduction des éponymes médicaux banalisés de langue anglaise
}

\author{
HENRI VAN HOOF \\ Institut libre M. Haps, Bruxelles, Belgique
}

Par éponyme banalisé, il faut entendre tout terme substantivé (parkinsonism / parkinsonisme), verbalisé (to pasteurize / pasteuriser) ou adjectivé (parkinsonian / parkinsonien), par opposition à l'éponyme resté nom propre (Parkinson's syndromel maladie de Parkinson). Ces noms communs, bien que relativement peu nombreux comparés à la pléthore des noms propres, peuvent poser problème au traducteur pour de multiples raisons.

Observons tout d'abord que le langage médical, selon qu'il est anglais ou français, n'a pas toujours procédé aux mêmes banalisations. Ainsi, les substantifs anglais benneroma, dakinization, hahnemannism, highmoritis, kocherization, mansonelliasis, mullerianosis, rombergism n'ont pas d'équivalents de même nature en français; à l'inverse, l'anglais n'a pas substantivé le français «basedowisme aigu», «bauhinite», «curiepuncture», «eschérichiose», "hanséniase», «kupfférome», «rickettsie». De même, le français n'a pas adjectivé l'anglais gartnerian, glissonian, nabothian, pacchionian, pacinian, rickettsial, spigelian, tandis que l'anglais ne possède pas d'adjectifs correspondant au français «bravaisien», «hissien», «hodgkinien», «klinefeltérien», «korsakovien», «leydigien», "méniérique», "nougarien», "pagétique», «pottique», «ténonien", «vatérien», etc. On imagine donc aisément que la traduction de tous ces termes devra faire appel à une variété de procédés pour lesquels une analyse plus poussée ne sera pas inutile.

\section{Substantifs ${ }^{1}$}

Les problèmes liés à la traduction des substantifs proviennent de causes diverses : soit le français ne possède pas d'équivalent éponymique ou d'équivalent banalisé, soit il offre le choix entre deux formes banalisées ou, à l'inverse, n'en présente qu'une alors que l'anglais en a deux, soit il adjectivise le substantif ou vice versa, soit encore il se heurte à une divergence de terminaison, etc.

L'anglais dispose souvent, pour un même terme, de deux formes qui n'en appellent qu'une en français: bartonelliasis, bartonelosis / «bartonellose»; bilharziasis, bilharziosis / "bilharziose»; brownism, brunonianism / «brownisme»; brucelliasis, brucellosis / «brucellose»; lambliasis, lambliosis / «lambliase»; leishmaniasis, leishmaniosis / «leishmaniose»; meibomianitis, meibomitis / «meibomite»; nocardiasis, nocardiosis / "nocardiose», etc. On remarquera qu'il s'agit le plus souvent du doublet suffixal -iasis/-iosis et que le français préfère parfois l'un («lambliase»), parfois l'autre («bilharziose», «brucellose», etc.). D'autres problèmes de terminaison surgissent avec borreliotosis / "borréliose», eberthemia / "éberthite», rasorianism / «rasorisme». Parfois, c'est le français qui présente deux possibilités pour seulement une en anglais : miyagawanellosis / " miyagawanellose», "pararickettsiose»; cowperitis / «cowpérite», «mériyite»; odditis / "oddite», «sténose vatérienne bénigne», ou

Meta, XLVI, 1, 2001 
encore les deux termes français en partie différents des termes anglais: babesiasis, babesiosis / «babésiellose», «babésiose».

Dans certains cas, le français traduit par un éponyme banalisé l'éponyme resté nom propre en anglais: inflammation of Bauhin's valve / "bauhinite», Kupffer's cell sarcoma / «kupfférome», Rickett's organism / «rickettsie», etc. ou, à l'inverse, il faudra traduire par le nom propre de l'éponyme banalisé de l'anglais: brenneroma / «tumeur de Brenner», dakinization / "traitement par le liquide de Dakin», highmoritis / «antrite de Highmore», mansonelliasis / «infestation par la filaire Mansoni ozzardi », rombergism / «signe de Romberg», etc. Dans d'autres cas encore, le français recourt à d'autres éponymes que l'anglais: Bedsonia infection / «néorickettsiose», kocherization / «incision duodénale pour aborder l'ampoule de Vater», teslaization / «darsonvalisation».

Mais il arrive aussi que le français se passe complètement d'éponyme: «hahnemannism / «homéopathie»; mullerianosis, mulleriosis / «endométriose»; etc. ou qu'il introduise un éponyme où l'anglais n'en a pas: thyroid crisis / «basedowisme aigu», green blindness / "daltonisme deutéranope», colibacillosis / "eschérichiose», chlamydiosis / "pararickettsiose», middle cerebral artery syndrome / «syndrome de la sylvienne», etc.

\section{Verbes et formes verbales}

L'anglais a la possibilité de former des verbes en ajoutant le suffixe -ize à n'importe quel radical ou en verbalisant tout simplement le substantif. Ce n'est que très rarement que, dans le cas d'éponymes ainsi verbalisés, la traduction directe sera permise: to pasteurize / "pasteuriser», mais to faradize / "traiter par courant faradique», to haffkinize / "immuniser au sérum de Haffkine», to roentgenize / "traiter par les rayons $\mathrm{X}$ », to zenkerize / "fixer au liquide de Zenker»; à plus forte raison lorsqu'il s'agit de formes verbales: gatched-up position / "position demi-assise dans le lit de Gatch».

\section{Adjectifs}

Comme pour les substantifs, les problèmes soulevés par la traduction des adjectifs sont d'origines multiples: soit le français ne dispose pas d'équivalent éponymique ou d'équivalent banalisé, soit il traduit l'adjectif par le nom propre ou, à l'inverse, adjectivise le nom propre de l'anglais, soit il offre le choix entre deux formes banalisées pour la forme unique de l'anglais, soit il use d'un éponyme banalisé différent de l'anglais, soit encore il se trouve devant des différences de terminaison.

Ainsi, le français peut-il présenter, pour un même terme anglais, deux formes différentes: basedowified / "basedowifié», "basedowifiant»; bravais-jacksonian / «bravaisienne», "bravais-jacksonienne»; skodaic resonance / "bruit skodique», «skodisme»; etc. ou n'en possède-t-il qu'une pour deux formes anglaises: brownian, brunonian / "brownien», ou encore use-t-il d'un éponyme différent: gartnerian cyst / "kyste wolffien». On aura noté au passage qu'un adjectif banalisé est parfois rendu par le substantif banalisé (skodic resonance / skodisme); il en est encore ainsi dans inflammation of the haversian canals / "haversite», rickettsial pox / «rickettsiose varicelliforme». On aura noté aussi que les terminaisons adjectivales 
peuvent différer (basedowified / «basedowifiant»), comme c'est encore le cas pour basedoid / «basedowoïde»; bilharzial, bilharzic / «bilharzien»; brucellar / «brucellien»; hurloid / "hurlérien»; neisserial / «neisserien»; etc.

Dans de très nombreux cas, le français conserve le nom propre où l'anglais a adjectivé l'éponyme: darwinian ear / "oreille de Darwin», fallopian canal / «canal de Fallope», fallopian ligament / «ligament de Fallope», fallopian tube / «trompe de Fallope», gasserian ganglion / "ganglion de Gasser», haversian canals / "canaux de Havers", meibomian glands / "glandes de Meibom», mullerian duct / "canal de Müller», nabothian cysts / "œufs de Naboth», pacchionian bodies / "granulations de Pacchioni», pacinian corpuscles / "corpuscules de Pacini», rolandic fissure / «sillon de Rolando», ruyschian membrane / "membrane de Ruysch», spigelian hernia / "hernie de Spigel», sylvian fissure / "scissure de Sylvius», wolffian body / "corps de Wolff», etc. Il n'en faudrait toutefois pas conclure qu'aucun de ces adjectifs n'existe en français car, paradoxalement, on trouve angular impression of gasserian ganglion / "fossette gassérienne», haversian system / "système haversien », «meibomian sty» / «acné meibomienne», mullerian cyst / «kyste mullérien», rolandic area / "aire rolandique», rolandic epilepsy / "épilepsie rolandique», sylvian artery / "artère sylvienne», wolffian tubules / «canalicules wolffiens», etc.

Tout aussi nombreux sont les cas où le français adjectivise le nom propre anglais: His bundle tachycardia / "tachycardie hissienne», non-Hodgkin lymphoma / "lymphome non hodgkinien", pseudo-Klinefelter syndrome / "syndrome klinefeltérien", Korsakoff's contact potencies / "dilutions korsakoviennes", Leydig-cell tumor / "tumeur leydigienne», Ménière's disease / "vertige méniérique», Nougaret night blindness / "héméralopie nougarienne », Paget's disease of bone / "ostéose pagétique ", Parkinson's facies / "faciès parkinsonien", Pott's paralysis / "paralysie pottique», Vater's ampulla carcinoma / "ampullome vatérien", dyskinesia of Wilson's disease / «dyskinésie wilsonienne», Zenker's degeneration / "dégénérescence zenkérienne», etc. On chercherait vainement une logique dans toutes ces créations éponymiques lorsqu'on voit coexister Ruysch's veins / "veines ruyschiennes» à côté de ruyschian membrane / "membrane de Ruysch", cistern of Sylvius / "confluent sylvien" à côté de sylvian fissure / "scissure de Sylvius», etc.

Enfin, il n'est pas rare que le français renonce à l'éponyme, comme dans fallopian pregnancy / "grossesse tubaire», glissonian cirrhosis / "cirrhose capsulaire", meibomian cyst / "chalazion», roentgen sickness / "mal des rayons», etc. ou, à l'inverse, qu'il introduise un éponyme là où l'anglais n'en a pas: thyroid heart / «cœur basedowien", pseudohyperthyroidism / «syndrome parabasedowien», albuminuric retinitis / "rétinite brightique», swelled head / "crâne pagétique», writhing hand / «main parkinsonienne», etc.

\section{Conclusion}

Ces quelques considérations, bien que limitées aux éponymes banalisés, illustrent bien la confusion qui règne dans le domaine de l'éponymie médicale, confusion dont se plaignent les médecins eux-mêmes et, faut-il s'en étonner, les étudiants en médecine qui doivent acquérir quelque 50000 mots au cours de leurs études. Le traducteur médical ne peut que se joindre à ce chœur de lamentations mais, placé devant la réalité du problème, il ne peut y faire face qu'en s'aidant d'une documentation adé- 
quate et par la fréquentation régulière et intensive de la littérature médicale dans les deux langues.

\section{NOTE}

1. Nous ne nous attarderons pas aux noms latins de bactéries et bacilles basés sur une éponymie simple (Salmonella typhi) ou double (Salmonella hirschfeldi), qui demeurent fondamentalement inchangés dans les deux langues, sauf à présenter parfois quelque forme supplémentaire dans l'une d'elles (Haemophilus ducreyi / Coccobacillus ducreyi, Haemophilus ducreyi).

\section{RÉFÉRENCES}

Agnew, L. R. C. et al. (1985): Dorland's Illustrated Medical Dictionary, Philadelphia, W. B. Saunders.

Chaumuzeau, J.-P. et al. (1975) : Dictionnaire de médecine Flammarion, Paris, Flammarion.

Delamare, J. et T. (1986) : Dictionnaire français-anglais/anglais-français des termes techniques de médecine, Paris, Maloine.

Gladstone, W. J. (1984): Dictionnaire anglais-français des sciences médicales et paramédicales, Paris, Maloine.

Thomas, C. L. et al. (1973) : Taber's Cyclopaedic Medical Dictionary, Philadelphia, F. A. Davis Co.

Van Hoof, H. (1993): Dictionnaire des éponymes médicaux français-anglais, Louvain-la-Neuve, Peeters.

\section{ANNEXE}

\section{Lexique}

addisonian (adj): addisonien addisonian crisis: crise addisonienne

addisonism: addisonisme

arsonvalism, arsonvalization: (d')arsonvalisation

Babesia, Babesiella: Babesia

babesiasis, babesiosis: babésiellose, babésiose

bancroftian (adj) : -

bancroftian filariasis: bancroftose

bancroftosis: bancroftose

bartholinitis : bartholonite

Bartonella: Bartonella

bartonelliasis, bartonellosis: bartonellose

basedoid (adj) : basedowoïde

basedowian (adj): basedowien

cardiothyreotoxicosis: cœur basedowien

pretibial pyxedema: dermopathie basedowienne

pseudohyperthyroidism: syndrome parabasedowien

tachycardia strumosa exophtalmia: tachycardie basedowienne

basedowified (adj) : basedowifié, basedowifiant

basedowified goiter: goitre basedowifiant

basedowiform (adj) : basedowiforme

-: basedowisme

thyroid crisis: basedowisme aigu

inflammation of Bauhin's valve: bauhinite

Bedsonia, Miyagawanella: Miyagawanella

Bedsonia infection: néorickettsiose

—: biermérien (adj)

Addison-Biermer's anemia with a neurologic onset: neuropathie achylique biermérienne 


\section{MeTA, XLVI, 1, 2001}

Bilharzia: Bilharzia

bilharziasis, bilharziosis : bilharziose

Bordetella: Bordetella

Borrelia: Borrelia

borreliotosis : borréliose

relapsing fever: borréliose récurrente

borreliota : borréliota

botallism: botallisme

braidism : braidisme

bravais-jacksonian (adj): bravaisienne, bravais-jacksonienne

bravais-jacksonian epilepsy: épilepsie bravaisienne/bravais-jacksonienne

brenneroma: tumeur de Brenner

brightic (adj) : brightique

albuminuric retinitis : rétinite brightique

brightism : brightisme

broussaisism: broussaisisme

brownian, brunonian (adj) : brownien

brownian/brunonian movement: mouvement brownien

brownism, brunonianism : brownisme

Brucella: Brucella

brucellar (adj) : brucellien

brucelliasis, brucellosis : brucellose

neurobrucellosis : neurobrucellose

buckytherapy: buckythérapie

chagoma: chagome

cowperitis: cowpérite, méryite

pericowperitis : péricowpérite

Craigia: Craigia

curie: curie

interstitial irradiation: curiepuncture

curietherapy: curiethérapie

cushingoid (adj): cushingoïde

dakinization: traitement par le liquide de Dakin

daltonian (adj) : daltonien

daltonism: daltonisme

green blindness: daltonisme deutéranope

smell blindness: daltonisme olfactif

darsonvalization (cf. arsonvalism)

darwinian (adj) : darwinien

darwinian ear: oreille de Darwin

darwinism: darwinisme

neodarwinism: néodarwinisme

Davainea: Davainea

descemetitis: descemétite

descemetocele: descemétocèle

donovanosis: donovanose

douglasitis: douglassite

Duttonella: Duttonella

Eberthella: Eberthella

eberthemia: éberthite

eberthian (adj): éberthien

Eimeria: Eimeria

Escherichia: Escherichia

colibacillosis: eschérichiose

esmarch : esmarch 


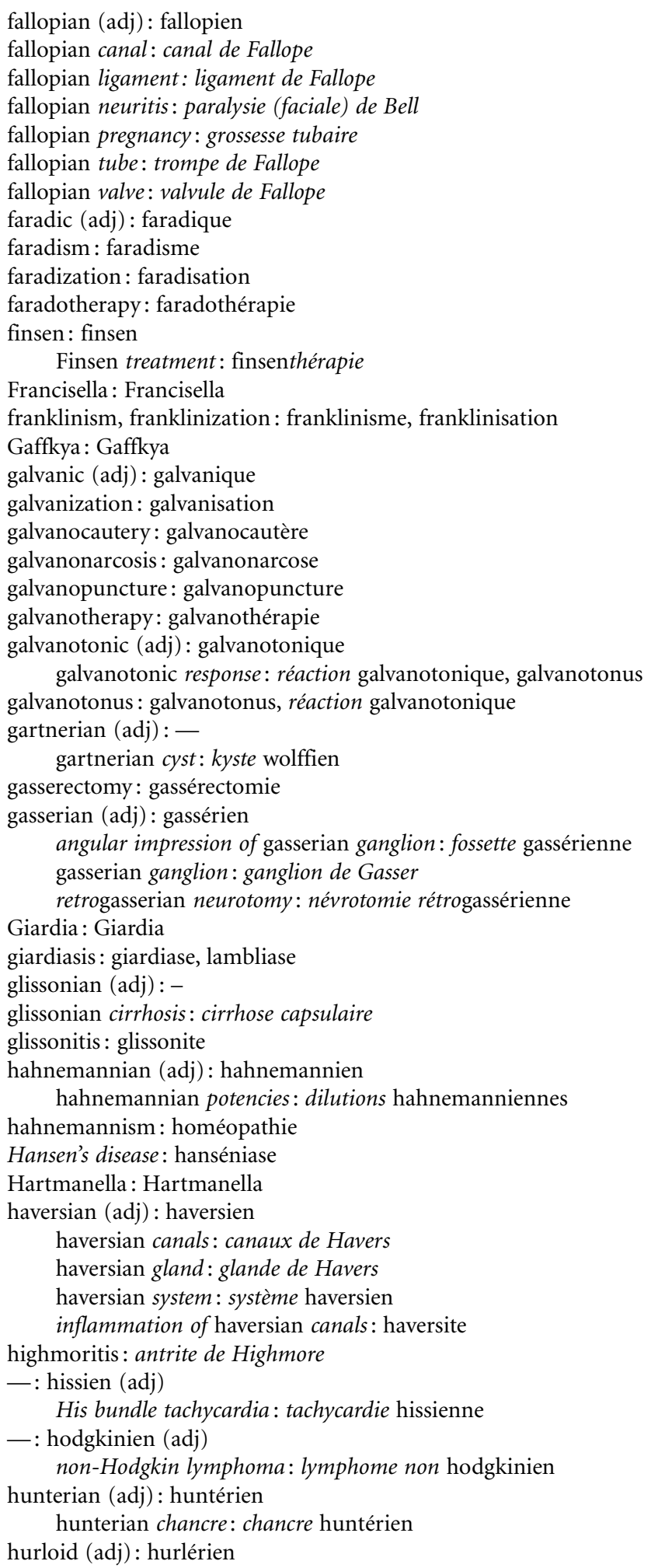


jacksonian (adj) : cf. bravais-jacksonian

jacksonian crisis: cf. bravais-jacksonian epilepsy

jacksonian epilepsy: cf. bravais-jacksonian epilepsy

jacksonian gait: marche jacksonienne

jennerian (adj): jennérien

jennerian vaccine: vaccination jennérienne

jennerization: jennérisation

Klebsiella : Klebsiella

—: klinefeltérien (adj)

pseudo-Klinefelter syndrome: syndrome klinefeltérien

kocherization: incision duodénale pour aborder l'ampoule de Vater

—: korsakovien (adj)

Korsakoff's contact potencies: dilutions korsakoviennes

- : kupfférien (adj)

Kupffer's cell sarcoma: kupfférome

Kurthia: Kurthia

lamarckism : lamarckisme

Lamblia: Lamblia

lambliasis, lambliosis: cf. giardiasis

langerhansian (adj) : langerhansien

langerhansian adenoma: adénome langerhansien

langerhansian hormone: hormone langerhansienne

- : leydigien (adj)

Leydig-cell function: fonction leydigienne

Leydig-cell tumor: tumeur leydigienne

Leishmania: Leishmania

leishmaniasis, leishmaniosis: leishmaniose

cutaneous leishmaniasis: leishmaniose cutanée

infantile leishmaniasis: leishmaniose splénique infantile

visceral leishmaniasis: leishmaniose viscérale

leishmanid, leishmanoid: leishmanide

lewisite: léwisite

Listerella, Listeria: Listeria

listerellosis, listeriosis : listérellose, listériose

littritis : littrite

lobomycosis: lobomycose

malpighian (adj) : malpighien

malpighian layer: assises malpighiennes

mansonellosis : infestation par la filaire Mansoni ozzardi

meibomian (adj): meibomien

meibomian cyst: chalazion

meibomian glands: glandes de Meibom(ius)

meibomian sty: acné meibomienne

meibomianitis, meibomitis: meibomite

—: méniérique (adj)

Ménière's disease: vertige méniérique

miyagawanellosis: miyagawanellose, pararickettsiose

Moraxella: Moraxella

morgagnian (adj): margagnien

morgagnian cataract: cataracte morgagnienne

mullerian: mullérien

antimullerian hormone: hormone antimullérienne

mullerian anomalies: anomalies mullériennes

mullerian cyst: kyste mullérien

mullerian duct: canal de Müller 
mullerianosis, mulleriosis: endométriose

mulleroblastoma: mulléroblastome

nabothian (adj) :-

nabothian cysts: oufs de Naboth

Neisseria: Neisseria

neisserial (adj) : neissérien

Nocardia: Nocardia

nocardiasis, nocardiosis: nocardiose

—: nougarien (adj)

Nougaret night blindness: héméralopie nougarienne

odditis: oddite, sténose vatérienne bénigne

pacchionian (adj) :

pacchionian bodies: granulations de Pacchioni

pacinian (adj):-

pacinian corpuscles: corpuscule de Pacini

-: pagétique (adj)

Paget's disease of bone: ostéose pagétique

swelled head: crâne pagétique

pagetoid (adj): pagétoïde

parkinsonian (adj): parkinsonien

antiparkinsonian : antiparkinsonien

Parkinson's facies: faciès parkinsonien

parkinsionan syndrome: syndrome parkinsonien, parkinsonisme

Ramsay Hunt syndrome: syndrome parkinsonien juvénile

parkinsonian tremor: tremblement parkinsonien

writhing hand: main parkinsonienne

parkinsonism : parkinsonisme, syndrome parkinsonien

Pasteurella: Pasteurella

pasteurellosis : pasteurellose

pasteurization: pasteurisation

- : pastorien (adj)

vaccination with an emulsion of attenuated bacteria: vaccination pastorienne

pavlovian (adj) : pavlovien

—: pottique (adj)

Pott's paralysis: paralysie pottique

rasorianism : rasorisme

Rickettsia: Rickettsia

rickettsial (adj) :

rickettsial disease: rickettsiose

rickettsial pox: rickettsiose varicelliforme

rickettsemia : rickettsémie

Rickett's organism: rickettsie

rickettsiosis : rickettsiose

chlamydiosis: néorickettsiose, pararickettsiose

roentgen : roentgen

roentgen $(\mathrm{adj})$ : roentgénien

roentgen cachexia: cachexie roentgénienne

roentgen sickness: mal des rayons

roentgenization: roentgénisation

roentgenkymography: radiokymographie

roentgenogram: roentgenogramme

roentgenography: roentgenographie

roentgenology: roentgenologie

roentgenoscopy: roentgenoscopie

roentgen $(\mathrm{o})$ therapy: roentgenothérapie 
rolandic (adj) : rolandique

rolandic area : aire rolandique

rolandic artery: artère rolandique

rolandic convolution: circonvolutions rolandiques

rolandic epilepsy: épilepsie rolandique

rolandic fissure: sillon de Rolando

rolandic gelatinous substance: substance gélatineuse de Rolando

rolandic tubercle: turbercule cendré de Rolando

rombergism : signe de Romberg

ruyschian (adj): ruyschien

ruyschian membrane: membrane de Ruysch

Ruysch's veins: veines ruyschiennes

Salmonella: Salmonella

salmonellosis: salmonellose

schwannitis, schwannosis: schwannite, schwannose

schwannoma, schwannoglioma: schwannome, schwannogliome

multiple schwannoma: schwannomatose

Shigella: Shigella

shigellosis: shigellose

skenitis : skénite

skodaic (adj) : skodique

skodaic resonance: bruit skodique, skodisme

spigelian (adj) : -

spigelian arteries: artères du lobe de Spiegel

spigelian hernia: hernie de Spiegel

spigelian line: ligne semi-lunaire de Spiegel

spigelian lobe: lobe de Spiegel

sylvian (adj) : sylvien

sylvian aqueduct: aqueduc de Sylvius

sylvian aqueduct syndrome: syndrome sylvien

sylvian artery: artère sylvienne

cistern of Sylvius: confluent sylvien

sylvian fissure: scissure de Sylvius

sylvian fossa: fosse de Sylvius

sylvian line: ligne de Sylvius

sylvian vein: veine sylvienne superficielle

middle cerebral artery syndrome: syndrome de la sylvienne

—: ténonien (adj)

tenonitis : ténonite

teslaization: (d')arsonvalisation

Theileria: Theileria

theileriasis : theilériose

tydallization: tyndallisation

—: vatérien (adj)

Vater's ampulla carcinoma: ampullolome vatérien

Veillonella: Veillonella

vidian (adj) : vidien

vidian artery: artère vidienne

vidian canal: canal vidien

vidian nerve: nerf vidien

vidian neuralgia: syndrome du nerf vidien

vidian vein: veine vidienne

voltaic (adj) : voltaïque

voltaic test: épreuve voltaïque

voltaization: voltaïsation 
voltaism : voltaïsme

wallerian (adj): wallérien

wallerian degeneration: dégénérescence wallérienne

wallerian law: loi de Waller

whartonitis : whartonite

—: wilsonien (adj)

dyskinesia of Wilson's disease: dyskinésie wilsonnienne

wirsungography: wirsungographie

wolffian (adj) : wolffien

wolffian adenoma, wolffian duct carcinoma: épithélioma wolffien

wolffian body: corps de Wolff

wolffian cyst: kyste wolffien

wolffian duct: canal de Wolff

wolffian tubules: canalicules wolffiens

wormian (adj) : wormien

wormian bones: os wormiens

Wuchereria: Wuchereria

wucheriasis: wuchériose

Yersinia: Yersinia

yersinosis: yersinose

—: zenkérien (adj)

Zenker's degeneration: dégénérescence zenkérienne

zenkerism : cf. ci-dessus

Zuberella: Zuberella 